\title{
Post-yield characterisation of metals with significant pile-up through spherical indentations
}

\author{
H. Habbab ${ }^{\text {a }}$, B.G. Mellor ${ }^{\text {b }}$, S. Syngellakis ${ }^{b, *}$ \\ ${ }^{a}$ KW Ltd., Fetcham Park House, Lower Road, Fetcham, Leatherhead, Surrey KT22 9HD, UK \\ ${ }^{\mathrm{b}}$ School of Engineering Sciences, Highfield, University of Southampton, Southampton, Hampshire SO17 1BJ, UK
}

Received 30 November 2005; received in revised form 16 December 2005; accepted 17 December 2005

Available online 21 February 2006

\begin{abstract}
Finite element simulations of spherical indentations accounting for frictional contact provide validated load-indentation output for assessing and improving existing methods used to determine the stress-strain curve of materials with significant pile-up. The importance of friction to the proper assessment of the pile-up effect is established. Weaknesses in current characterisation relations and procedures are also identified. Existing correction formulae accounting for pile-up are modified so that the contact area radius is more accurately determined. This modification is implemented in the context of a characterisation process that relies on analysing unloading portions of load-indentation curves. Post-yield material behaviour predictions from such analysis are found to be in very good agreement with the initial finite element material input.
\end{abstract}

(C) 2006 Acta Materialia Inc. Published by Elsevier Ltd. All rights reserved.

Keywords: Plastic deformation; Macroindentation; Finite element analysis; Steels; Stress-strain relation

\section{Introduction}

Instrumented indentation testing has been widely used as an attractive means of estimating material mechanical properties. As the hardness resulting from a spherical indenter increases with penetration depth to an extent dependent on the degree of strain hardening of the material, the post-yield stress-strain curve of a ductile material can be determined by carrying out a series of hardness tests at different loads. According to Tabor's original method [1], the evaluation of both flow stress and the corresponding, so-called, representative strain relies on knowledge of the contact area. Advances in high-resolution measurement instruments have facilitated probing the material surface on the micrometre scale using a very small indenter. On such a scale, it is a tedious and laborious task to determine

\footnotetext{
* Corresponding author. Tel.: +44 2380592844; fax: +44 2380594813.

E-mail address: ss@soton.ac.uk (S. Syngellakis).
}

the contact area through optical measurement bearing in mind the uncertainty in defining the contact edge of a residual indent. Thus a simple approach [2] has been proposed to extract post-yield material behaviour from cyclic loadindentation data. This consists of determining the contact depth at maximum load over each load cycle. Analysis of the unloading portion of the load-indentation curve, along which the behaviour of the material is considered elastic and hence governed by the Hertz contact solution, leads to the projected contact area.

The effectiveness of this technique relies heavily on the assumption that the contact edges deform in the direction of the indenter movement, that is, the material deforms below the original surface sinking-in. Whereas this represents the behaviour of hard materials, soft metals act differently in that the contact perimeter tends to rise above the level of the original surface forming a raised lip surrounding the impression. This phenomenon is known as pile-up. A schematic illustrating the geometry of an indent in both cases is shown in Fig. 1 where a dotted line represents the permanent indent profile after unloading. 


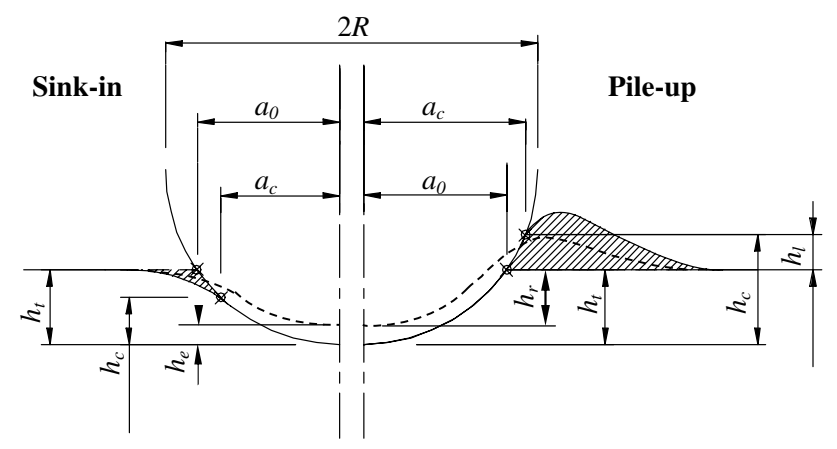

Fig. 1. A schematic illustration of piling up and sinking in associated with spherical indentations.

Ignoring the pile-up effect results in an error in estimating the contact area, which was found to be as much as $60 \%$ for conical indentations on aluminium [3]. Empirical correction formulae were proposed [4-6] to account for the pile-up effect assuming a priori knowledge of the strain hardening behaviour of the material. This knowledge is, however, one of the objectives of a complete characterisation process.

In this paper, an iterative scheme is devised for including the pile-up effect in Tabor's original characterisation procedure using established correction formulae. The performance of the proposed method is assessed by applying it to simulated spherical indentations on ideal materials, with known post-yield behaviour characterised by a strain hardening exponent and a strength coefficient. A serious weakness in the existing pile-up correction formulae is identified, which is then addressed by introducing into these formulae an empirical factor based on finite element estimates of the contact area.

\section{Finite element model and materials}

As pointed out in Section 1, established characterisation procedures are here tested by applying them to simulated indentation records obtained from a validated finite element analysis. The elastic-plastic model developed was based on ANSYS, a general-purpose finite element package [7]. The problem was solved as axisymmetric, and thus only half of the indenter and the material cylinder sectioned by a plane through the axis of symmetry was modelled. Isotropic hardening plasticity and the von Mises yield criterion were assumed. Large strain and other non-linear features of ANSYS were incorporated in the model. The spherical indenter was modelled as elastic with a modulus of elasticity $E=1140 \mathrm{GPa}$, Poisson's ratio $v=0.07$, and radius $R=150 \mu \mathrm{m}$.

The model areas were meshed with four-node quadrilateral elements while surface-to-surface contact was modelled through a pair of contact and target elements. The analysis was initially applied to frictionless elastic indentation so that the results could be compared with the corresponding Sneddon's solution [8]. These comparisons allowed a sensitivity study on mesh size and density to be carried out leading to the selection of the mesh shown in

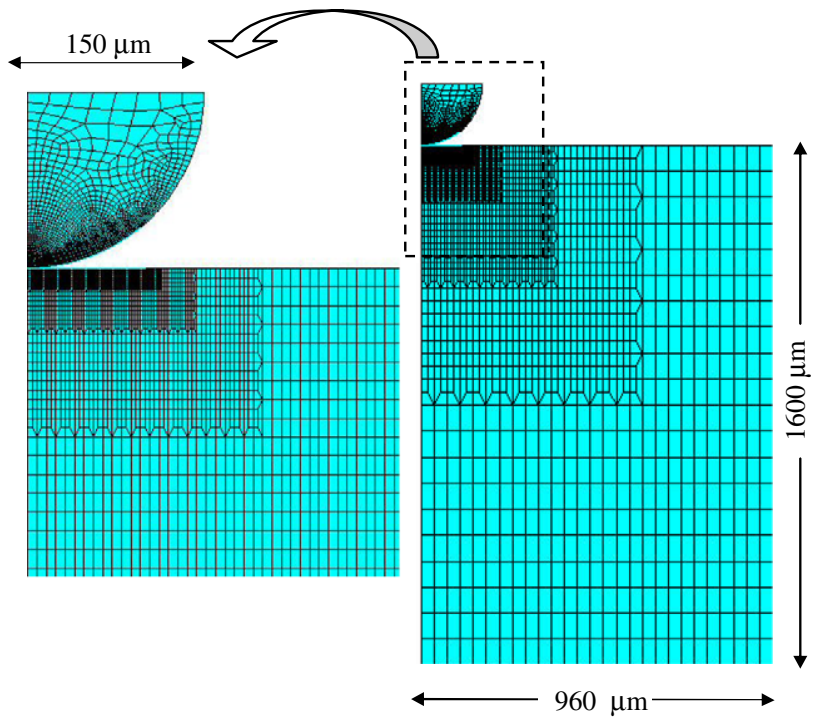

Fig. 2. Final mesh adopted in finite element simulations of spherical indentation.

Fig. 2 for which agreement between exact analytical and finite element results was excellent.

The elastoplastic indentation simulations were carried out on representative structural steel materials whose post-yield portion of the stress-strain curve can be simplified to and idealised as a single power law function of the form

$\sigma=K \varepsilon^{n}$

where $\sigma$ is the true stress, $\varepsilon$ is the logarithmic strain, and $n$ is the strain hardening exponent. $K$ is the strength coefficient and is determined from satisfying the continuity condition at yield point:

$\varepsilon_{0.2}=0.002+\frac{\sigma_{0.2}}{E}$

The input parameters of the materials idealised as above are summarised in Table 1.

The choice of idealised materials was made to cover two grades of structural steels referred to as $\mathrm{S} 3$ and $\mathrm{S} 5$, in addition to austenitic stainless steel, SS. The elastic moduli were taken equal to 210 and $185 \mathrm{GPa}$ for structural and stainless steel, respectively. The loading and unloading was modelled as prescribed displacement. The loading pattern was either in one cycle or in multiple cycles at one location, where unloading takes place at the peak load of each cycle followed by progressively increasing load in the subsequent cycles.

Table 1

Post-yield material parameters

\begin{tabular}{llll}
\hline Post-yield parameter & S3 & S5 & SS \\
\hline$n$ & 0.190 & 0.132 & 0.250 \\
$K(\mathrm{MPa})$ & 745 & 783 & 1168 \\
$\sigma_{0.2}(\mathrm{MPa})$ & 250 & 375 & 285 \\
\hline
\end{tabular}




\section{Consistency between finite element output and characterisation relations}

For an ideal plastic material, Tabor [9] has correlated the mean pressure or Meyer hardness $p_{\mathrm{m}}$ to the yield stress in simple compression $\sigma_{\mathrm{Y}}$ through the constraint factor $C$, which was empirically found to be approximately equal to 3 for steel. For strain hardening materials, the yield stress is replaced by the true flow stress $\sigma_{\mathrm{r}}$ corresponding to the representative strain, $\varepsilon_{\mathrm{r}}$. Thus, their relation has the form

$p_{\mathrm{m}}=C \sigma_{\mathrm{r}}$

while $\varepsilon_{\mathrm{r}}$ is obtained from

$\varepsilon_{\mathrm{r}}=0.2 \sin \beta=0.2 \frac{a_{\mathrm{c}}}{R}$

where $a_{\mathrm{c}}$ is the projected contact area radius and $\beta$ the angle between the tangent to the indenter at the contact edge and the original surface. Since Meyer's hardness is given by

$p_{\mathrm{m}}=\frac{P}{\pi a_{\mathrm{c}}^{2}}$

where $P$ is the indentation load, it is important that $a_{\mathrm{c}}$ is accurately determined for a reliable post-yield characterisation.

In order to verify that the finite element modelling results are consistent with the fundamental principle linking true stress to logarithmic strain, the contact area was determined by direct measurement from the simulated indentation profile at every load step. Thus, the gradual change in the predicted value of $a_{\mathrm{c}}$ with load was monitored. Due to the discrete nature of the finite element solution, the contact radius obtained from the indentation profile is always an underestimate since the contact edge, defining the contact area radius, can only be detected at the furthest specimen node in contact with the indenter. This results in a non-smooth variation of the estimated Meyer hardness manifested in the oscillatory plot of hardness versus indentation strain shown in Fig. 3. It is therefore rational to adopt as accurate only the lower bound values of hardness, forming the smooth solid curve shown in Fig. 3, corresponding to more reliable estimates of the contact radius $a_{\mathrm{c}}$.

Since Tabor's Eqs. (3)-(5) were based on observation of indentations on real materials, it was considered important at this stage to include friction between indenter and specimen in the finite element modelling. This can be achieved in ANSYS by specifying a contact friction coefficient, which was given values of $0,0.05,0.1,0.2$, and 0.5 . With the simulation applied to material $\mathrm{S} 5$, the resulting load-indentation depth $(P-h)$ curves started to deviate from each other at around two-thirds of a maximum depth of $25 \mu \mathrm{m}$, while the difference at the peak load was less than $4 \%$. This difference is highlighted in Fig. 4. The results from the other two materials showed the same trend.

The effect of friction on the resulting $P-h$ curves thus appears to be negligible. However, when the profiles of

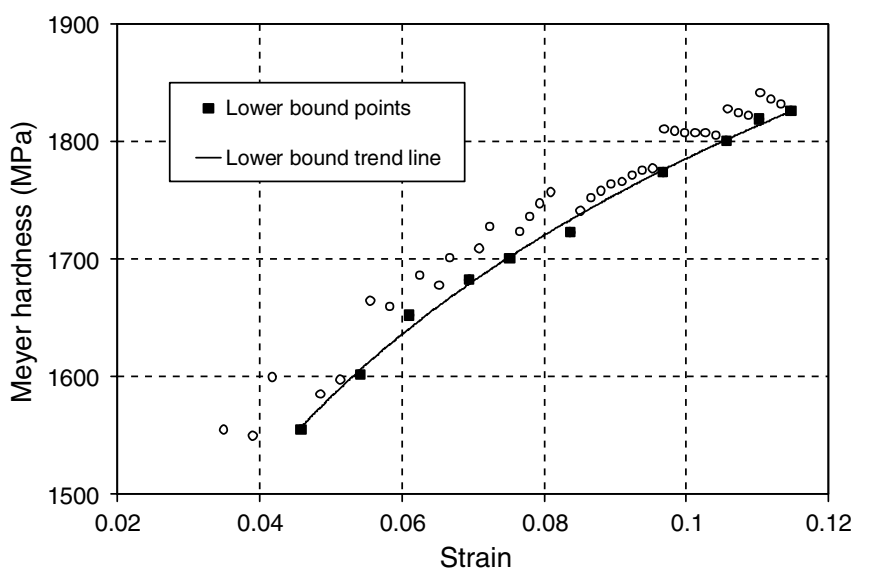

Fig. 3. Meyer hardness variation based on $a_{\mathrm{c}}$ extracted from the simulated indentation profile.

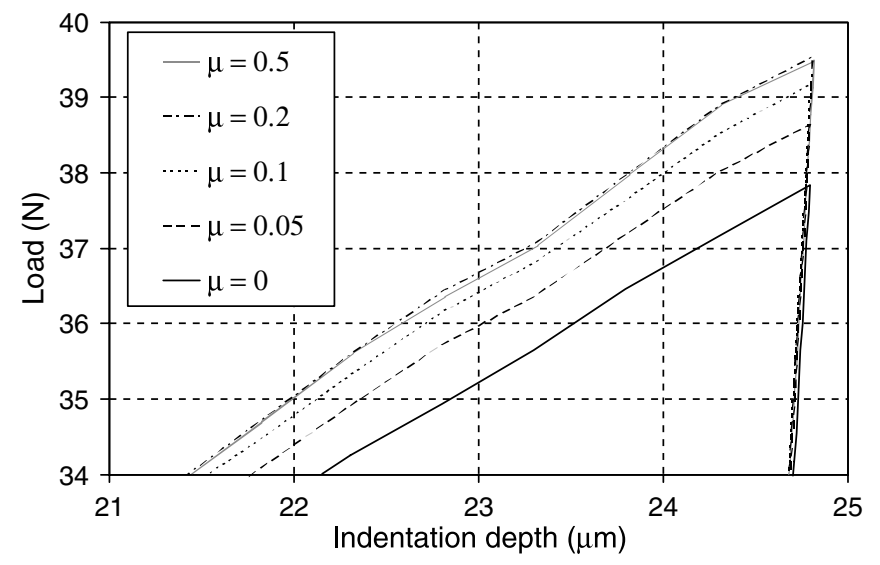

Fig. 4. Highlighted portion of finite element-simulated load-indentation curves for a range of friction coefficients.

the edge of the indent at peak load for the five friction coefficients are compared in Fig. 5, the height of the pile-up above the original specimen surface is seen to drop by over $50 \%$ when the friction coefficient increases from 0 to 0.5 . The influence of friction coefficient on contact depth and

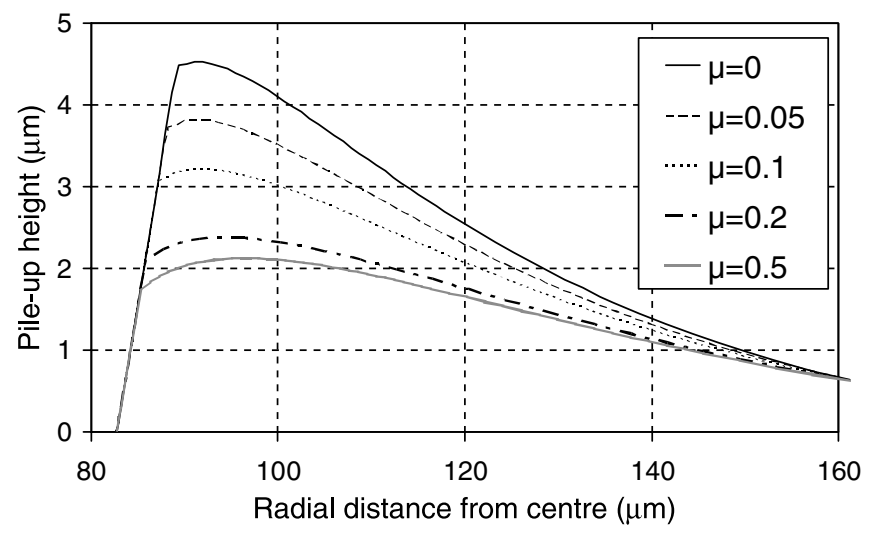

Fig. 5. Edge of indentation profiles at peak load for a range of friction coefficients. 
area is therefore found to be significant, in agreement with other recently reported results [10].

The direct measurement of $a_{\mathrm{c}}$ from the simulated indentation profiles at various load levels was obtained for several values of the friction coefficient $\mu$. For each $\mu$ value, the post-yield stress-strain curve was calculated according to Tabor's method, Eqs. (3)-(5), assuming $C=3$. These predictions can be compared with the original finite element input curve for S5 by referring to Fig. 6 . The plot also includes the values of $n$,obtained as the gradient of the line fitted to the respective hardness versus strain plots on a logarithmic scale. In addition, the resulting $0.2 \%$ yield stress $\sigma_{0.2}$ for each of the stress-strain curves together with the corresponding strain hardening exponent $n$ and strength coefficient $K$ are presented in Table 2.

The analysis results indicate a strong effect of contact friction on the calculated stress-strain curve. A value of $\mu$ around 0.13 appears to give the best agreement between the predicted and input $\sigma-\varepsilon$ curves. By comparing the results of Table 1 with the data for S5 in Table 1, $\mu=0.13$ is also seen to give excellent agreement between original and predicted strain hardening exponent, strength coefficient, and $\sigma_{0.2}$. This outcome is consistent with experimental findings [11] regarding sliding friction of diamond on steel. It may also be noted that $\mu=0.1$ is a widely accepted value for frictional contact between metal and diamond $[10,12,13]$. Thus, $\mu=0.13$ is adopted as the optimum value to represent the real friction developing between the two bodies during indentation and all further finite element results were obtained using this value.

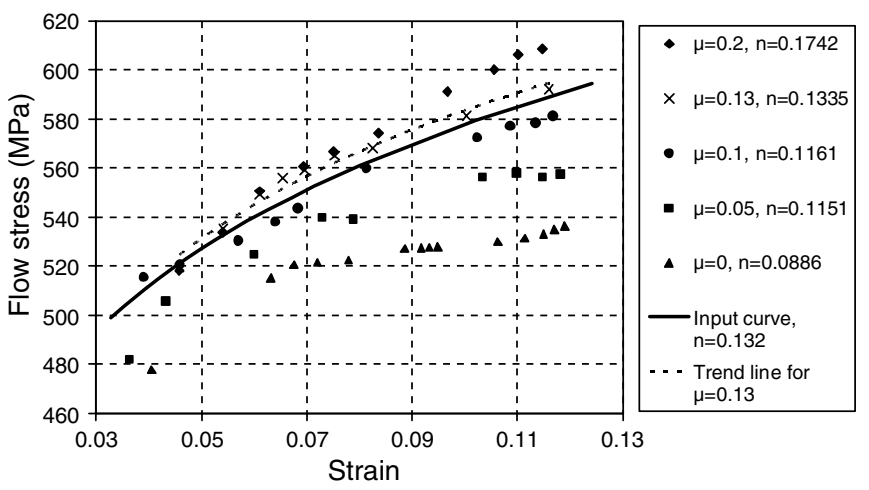

Fig. 6. Stress-strain curves for S5 based on finite element predictions of contact radius for a range of friction coefficients.

Table 2

Material parameters for S5 based on finite element predictions of contact radius for various values of friction coefficient

\begin{tabular}{lllllll}
\hline & $\mu=0.0$ & $\mu=0.05$ & $\mu=0.10$ & $\mu=0.13$ & $\mu=0.20$ & $\mu=0.50$ \\
\hline$n$ & 0.09 & 0.12 & 0.12 & 0.13 & 0.17 & 0.18 \\
$K$ & 651 & 720 & 745 & 785 & 894 & 903 \\
$\begin{array}{l}(\mathrm{MPa}) \\
\sigma_{0.2}\end{array}$ & 398 & 379 & 391 & 373 & 335 & 329 \\
$(\mathrm{MPa})$ & & & & & & \\
\hline
\end{tabular}

\section{Characterisation based on elastic unloading}

The characterisation method proposed by Field and Swain $[2,14]$ is based on Tabor's work [1,9], supplemented by a model for extracting the indentation geometry from analysing the unloading portion of the $P-h$ curve based on Hertz's contact solution. According to this solution, the contact depth $h_{\mathrm{c}}$ is given by

$h_{\mathrm{c}}=\frac{h_{\mathrm{t}}+h_{\mathrm{r}}}{2}$

where $h_{\mathrm{r}}$ is the residual depth and $h_{\mathrm{t}}$ the depth from the original surface as shown in Fig. 1. The contact radius is then obtained from

$a_{\mathrm{c}}=\sqrt{2 R h_{\mathrm{c}}-h_{\mathrm{c}}^{2}}$

It should be pointed out that Eq. (6) is valid only when the material under the indenter sinks in, as illustrated in the left part of Fig. 1. The residual depth can be predicted by considering only two points along the unloading curve [14]

$P=\varphi\left(h-h_{\mathrm{r}}\right)^{1.5}$

where $\varphi$ depends on the geometry and elastic properties of the two bodies. Whilst this may give accurate results in the case of finite element-generated data, it loses reliability when applied to experimentally produced unloading curves due to the unavoidable noise included in such data. In the latter case, a more rational way of describing the whole unloading curve is by means of fitting the non-linear relation Eq. (8) to the initial portion of the unloading data.

This procedure was applied to the simulated unloading curves obtained through a cyclic loading pattern with progressively increasing cycle peaks. The simulation was carried out on the materials of Table 1 for a range of friction coefficient values. The stress-strain curves obtained for each friction level can be compared with the S5 input curve by referring to Fig. 7, while the corresponding material parameters, that is, $n, K$, and $\sigma_{0.2}$ are summarised in Table 3.

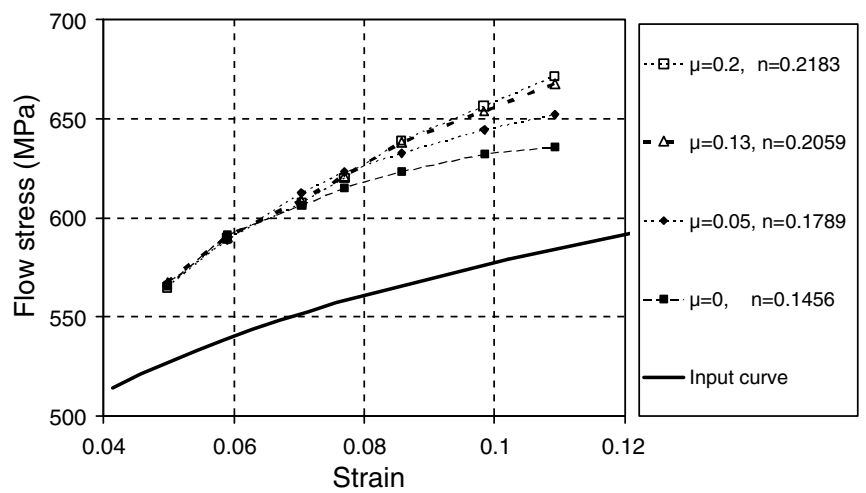

Fig. 7. Stress-strain curves for S5 by Field and Swain's method applied to simulated $P-h$ curves. 
Table 3

Material parameters for S5 from stress-strain relations obtained by Field and Swain's method

\begin{tabular}{lllllll}
\hline & $\mu=0.0$ & $\mu=0.05$ & $\mu=0.10$ & $\mu=0.13$ & $\mu=0.20$ & $\mu=0.50$ \\
\hline$n$ & 0.15 & 0.18 & 0.20 & 0.21 & 0.22 & 0.22 \\
$K$ & 887 & 978 & 1028 & 1054 & 1089 & 1089 \\
$(\mathrm{MPa})$ & & & & & & \\
$\begin{array}{c}\sigma_{0.2} \\
(\mathrm{MPa})\end{array}$ & 395 & 359 & 342 & 331 & 317 & 318 \\
\hline
\end{tabular}

It is interesting to note the strong influence of friction on the results of Fig. 7 and Table 3, although this characterisation method relies only on the unloading portion of the $P$ $h$ curves, which does not appear in Fig. 4 to be significantly affected by friction. Because of the ignoring of pile-up, the predicted $n$ is always greater than that of the input $\sigma-\varepsilon$ curve $(n=0.132)$, increasing with the value of $\mu$, whilst $\sigma_{0.2}$ shows an opposite trend. The calculated flow stress is overestimated by $10-17.5 \%$ compared with that of the input curve. Moreover, the effect of friction on the stressstrain curve obtained is noticeable when $\mu$ is less than 0.13 .

\section{Effect of neglecting pile-up on estimated contact depth}

The application of the Hertz solution to the unloading curve for materials exhibiting pile-up during indentation would result in underestimating the contact area leading to underestimation of the representative strains and overestimation of the Meyer hardness. As shown in the previous section, such predictions lead to incorrect estimates of all material parameters.

It has been conventionally accepted that the pile-up effect can be accounted for through the parameter $c^{2}$, the ratio of the contact depth to the total penetration depth from the original surface [15]:

$c^{2}=\frac{h_{\mathrm{c}}}{h_{\mathrm{t}}} \cong \frac{a_{\mathrm{c}}^{2}}{a_{\mathrm{o}}^{2}}$

where $a_{\mathrm{O}}$ is the radius of the section of the indenter by the original surface. It was also assumed that $c^{2}$ can be described as a unique function of $n$. Based on experimental measurements [15], various investigators have suggested empirical relations between $c^{2}$ and the $n$ value of the material that fit the experimental data.

Matthews [4] proposed an expression of the form

$c^{2}=\frac{1}{2}\left(\frac{2+n}{2}\right)^{2\left(\frac{1}{n}-1\right)}$

Hill et al. [16] suggested the following expression:

$c^{2}=\frac{5}{2}\left(\frac{2-n}{4+n}\right)$

Based on finite element simulations of indentations on many materials with different $n$, Taljat et al. [6] proposed two expressions for $c^{2}$ for fully loaded and unloaded indents:

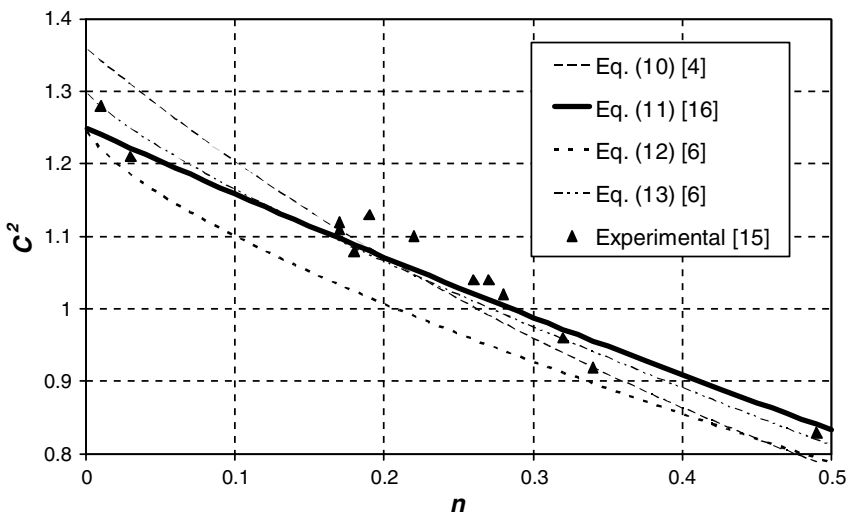

Fig. 8. Variation of correction factor $c^{2}$ with $n[4,6,16]$.

Loaded : $c^{2}=\frac{h_{\mathrm{c}}}{h_{\mathrm{t}}}=\frac{1}{4}\left(5-3 n^{0.7}\right)$

Unloaded $: c^{2}=\frac{h_{\mathrm{c}}}{h_{\mathrm{t}}}=\frac{1}{10}\left(13-8.5 n^{0.8}\right)$

All the expressions above except Eq. (13) yield the value of 0.5 when $n=1$, which coincides with the case of perfectly elastic behaviour, whilst Eq. (13) gives the value of 0.45 . A comparison of the predictions of the various expressions given above can be made by referring to Fig. 8 .

\section{Performance of the pile-up correction formulae}

In order to assess the accuracy of the various expressions for the correction factor $c^{2}$ mentioned in Section 5, the validation analysis presented in Section 4 is repeated here using the predicted $h_{\mathrm{t}}$ to compute $a_{\mathrm{c}}$ from each of the formulae of Eqs. (10)-(13). Results were again obtained for the S5 material, whose $n$, according to Table 1, is equal to 0.132. Comparison of the input $\sigma-\varepsilon$ curve for S5 with those obtained using the various pile-up corrections can be made by referring to the plots of Fig. 9, which also includes the $\sigma-\varepsilon$ curve resulting from the characterisation analysis of Section 4, corresponding to $\mu=0.13$. The

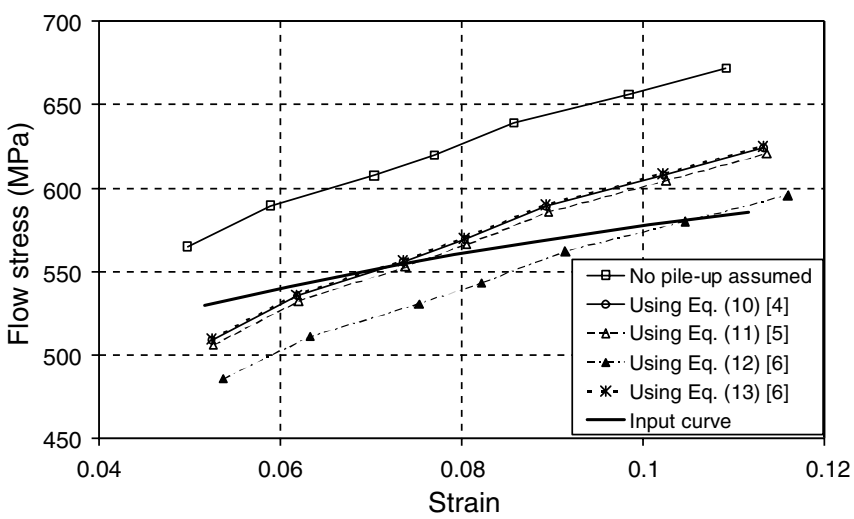

Fig. 9. Predicted stress-strain curves using the pile-up correction formulae. 
Table 4

Predictions of $n, K$, and $\sigma_{0.2}$ from simulated unloading curves using pile-up correction formulae (material S5, $\mu=0.13$ )

\begin{tabular}{llllll}
\hline & $\begin{array}{l}\text { Based on } \\
\text { Eq. }(10)\end{array}$ & $\begin{array}{l}\text { Based on } \\
\text { Eq. (11) }\end{array}$ & $\begin{array}{l}\text { Based on } \\
\text { Eq. (12) }\end{array}$ & $\begin{array}{l}\text { Based on } \\
\text { Eq. (13) }\end{array}$ & $\begin{array}{l}\text { No pile-up } \\
\text { correction }\end{array}$ \\
\hline$n$ & 0.26 & 0.26 & 0.26 & 0.26 & 0.20 \\
$K(\mathrm{MPa})$ & 1170 & 1145 & 1231 & 1159 & 1028 \\
$\sigma_{0.2}(\mathrm{MPa})$ & 259 & 253 & 274 & 257 & 342 \\
\hline
\end{tabular}

respective plasticity parameters $n, K$, and $\sigma_{0.2}$ are listed in Table 4.

Fig. 9 shows that, over the strain range considered, the validation analysis with the various pile-up corrections predicts flow stresses in better agreement with those of the original S5 input than the characterisation analysis, which ignores pile-up. However, according to Table 1, the values of the parameters $n$ and $K$ are much less accurate suggesting the need for a re-examination of the pile-up effect. It is interesting to note that the predicted value of $n$ is independent of the value of $c^{2}$ calculated from the various correction expressions. This can be easily shown to be the consequence of the adopted exponential form of the stress-stress relation, Eq. (1), the characterisation relations, Eqs. (3)-(5), as well as the fact that $c^{2}$ given by Eqs. (10)(13) depends only on $n$ and not on the indentation depth $h_{\mathrm{t}}$. This latter assumption is reassessed in Section 7.

Based on the requirement that the input value of $n$ should be equal to that obtained from the predicted stress-strain curve, an iterative process is suggested that obviates the need for prior knowledge of $n$. It starts with a valid arbitrary input of $n$, say 0 , to a correction formula for calculating a 'corrected' contact area and hence a flow stress-strain relation. Then the new value of $n$ obtained from this 'corrected' flow stress-strain relation is re-entered to the pile-up correction formula to recalculate a new, 'corrected' contact area. This iterative process terminates when two successive output values of $n$ are equal within a predefined tolerance.

Although the scheme is mathematically sound, it fails to yield results different from those obtained with a known value of $n$ because it reaches its target after only a single iteration. This is the consequence of $c^{2}$ assumed independent of $h_{\mathrm{t}}$ as explained above.

\section{Reassessment of the pile-up effect based on finite element predictions}

The conclusion drawn from the results of the previous section is that the pile-up correction formulae of Eqs. (10)-(13) fail to give a satisfactory prediction of the postyield stress-strain curve. In order to reassess the validity of these formulae, finite element predictions of the indentation profile during loading were employed to monitor the change of contact area and hence the development of pile-up by calculating its parameter, $c^{2}$, directly from the simulated profile at every load step. The resulting values for $c^{2}$ for each indentation depth can be compared to those

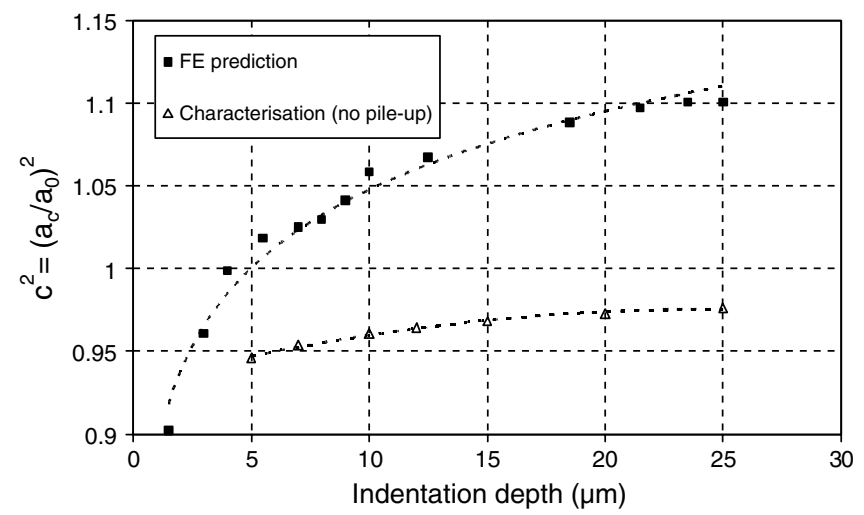

Fig. 10. Pile-up correction factor $c^{2}$ from the finite element-predicted indentation profile $(\mu=0.13)$.

calculated from the Field and Swain characterisation analysis that ignores pile-up by referring to Fig. 10 .

It is noted that the $c^{2}$ value from the indentation profile is not independent of the indentation depth as assumed by Eqs. (10)-(13). Initially, $c^{2}$ is less than unity for indentations less than $4 \mu \mathrm{m}$ deep, as pile-up has not yet started forming. When the indentation exceeds $5 \mu \mathrm{m}$ in depth, pile-up starts building up but at a decreasing rate, and then stabilises when indentation exceeds $20 \mu \mathrm{m}$ in depth. At this stage, the value of $c^{2}$ gets closer to that predicted by Eqs. (10)-(13). Fig. 10 also shows that the characterisation analysis by Field and Swain [2,14] predicts the $c^{2}$ dependence on the indentation depth. Although $c^{2}$ is always less than unity in this case since pile-up has been ignored, this result would be acceptable for hard materials that sink in. As a consequence, $c^{2}$ cannot always be considered independent of $h_{\mathrm{t}}$ and Eqs. (10)-(13) need to be modified for low and moderate indentations by a spherical indenter so that they account more accurately for the effect of pile-up, and hence predict the correct hardness and $n$ from such indentations.

\section{Modified modelling of the pile-up effect}

Finite element simulations show that the ratio $\left(a_{\mathrm{c}} / a_{\mathrm{o}}\right)^{2}$ is not constant as assumed by the expressions given by Eqs. (10)-(13). This suggests the need for modifying these expressions so that they account for the dependence of $\left(a_{\mathrm{c}} / a_{\mathrm{o}}\right)^{2}$ on indentation depth. Based on the same finite element output as that generating Fig. 10, the ratio of the residual depth $\left(h_{\mathrm{r}}\right)$ to the total indentation depth $\left(h_{\mathrm{t}}\right)$ was calculated and plotted against the total indentation depth in Fig. 11. It can be seen in that figure that the variation of $h_{\mathrm{r}} / h_{\mathrm{t}}$ follows the same trend as that of finite element-predicted $c^{2}$, which is shown in Fig. 10.

This observation suggests that a coefficient depending on the ratio $\left(h_{\mathrm{r}} / h_{\mathrm{t}}\right)$ needs to be appended to Eqs. (10)(13). The physical reason for this suggestion is the dependence of pile-up on the extent of plastic deformation, which can be considered directly linked to the magnitude of residual indentation. By examining the finite element indentation output for the three different materials, whose 


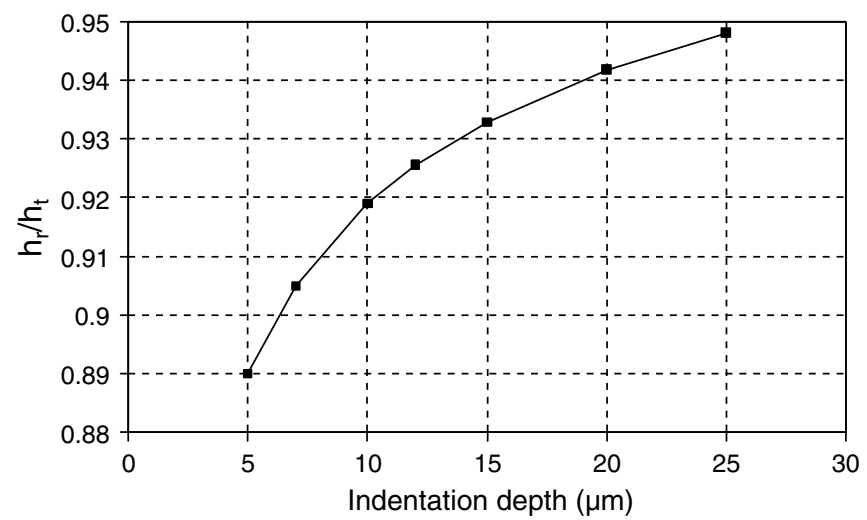

Fig. 11. Ratio of the residual to total indentation depth versus total indentation depth.

properties are listed in Table 1, a statistical study was carried out to correlate $\left(a_{\mathrm{c}} / a_{\mathrm{o}}\right)^{2}$ with $\left(h_{\mathrm{r}} / h_{\mathrm{t}}\right)$ and $c^{2}$ given by Eq. (11), proposed by Hill et al. [16]. This expression for the correction factor was chosen because it was based on measurement of real indentation profiles rather than finite element simulations of indentation of metals with a particular $E / \sigma_{\mathrm{Y}}$ ratio. In addition, Eq. (11) gives a better fit to experimental data than Eq. (10).

It was anticipated that the pile-up factor $\left(a_{\mathrm{c}} / a_{\mathrm{o}}\right)^{2}$ would be a product of $c^{2}$ and an expression depending on the ratio $h_{\mathrm{r}} / h_{\mathrm{t}}$. This expression should be equal to unity for purely elastic indentation, that is when $h_{\mathrm{r}}=0$, resulting in $\left(a_{\mathrm{c}} / a_{\mathrm{o}}\right)^{2}=0.5$, which complies with the elastic contact solution. Hence, it should also include a term depending on $h_{\mathrm{e}} / h_{\mathrm{t}}$, where $h_{\mathrm{e}}=h_{\mathrm{t}}-h_{\mathrm{r}}$ is the elastically recoverable indentation depth as shown in Fig. 1. Thus, the ratio $h_{\mathrm{e}} / h_{\mathrm{t}}$ should be equal to unity when $h_{\mathrm{r}} / h_{\mathrm{t}}=0$. After testing various simple functions for correlating the pile-up with the indentation data, a relation of the form

$$
\left(\frac{a_{\mathrm{c}}}{a_{\mathrm{o}}}\right)^{2}=c^{2} \sqrt{\gamma\left(\frac{h_{\mathrm{r}}}{h_{\mathrm{t}}}\right)^{z}+\left(\frac{h_{\mathrm{e}}}{h_{\mathrm{t}}}\right)^{z}}
$$

was shown to give better results in describing the finite element prediction presented in Fig. 10 for the materials modelled. These materials were chosen to cover the commonly encountered properties of structural steel. The strain hardening exponent and $0.2 \%$ yield stress for these materials range from 0.132 to 0.25 and from 250 to $375 \mathrm{MPa}$, respectively. By fitting the non-linear Eq. (14) to the finite element output for the three idealised steel specimens, the constants of this equation were determined as $z=3$ and $\gamma=1.125$. Thus, pile-up is accounted for through an empirical formula (Eq. (14)), proposed here for estimating the contact area, as a function of $n$ and $h_{\mathrm{r}} / h_{\mathrm{t}}$. As indicated in Section 4, the residual depth $h_{\mathrm{r}}$ can be determined from fitting the non-linear Eq. (8) to the unloading portion of the $P-h$ curve.

The pile-up correction parameter for the three materials, obtained from Eq. (14), can be compared to those extracted from the finite element-simulated indentation profiles by

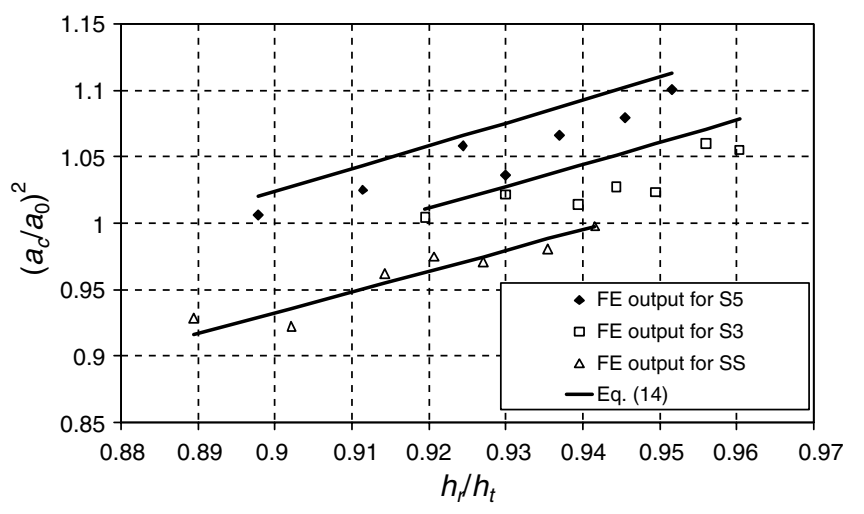

Fig. 12. Pile-up correction factor from Eq. (14) and simulated indentation profile versus $h_{\mathrm{r}} / h_{\mathrm{t}}$.

referring to Fig. 12, which shows $\left(a_{\mathrm{c}} / a_{\mathrm{o}}\right)^{2}$ as a function of $h_{\mathrm{r}} / h_{\mathrm{t}}$. As seen in Fig. 12, the prediction of the proposed Eq. (14) is in good agreement, within $1 \%$, with that obtained from finite element simulations of the indentation profile for the three materials. This confirms the validity of the non-linear fitting used to determine the constants in Eq. (14). The small difference observed is actually in favour of the accuracy of the proposed formula since the contact radius obtained from finite element modelling is always underestimated. Even though the derivation of this correction is based on data from three materials, its application to other steels within the same range of $n$ and $0.2 \% \sigma_{\mathrm{Y}}$ is expected to be valid. Furthermore, Eq. (14) has the capability of predicting the true contact area even when it lies beneath the original plane of the specimen surface, which happens during shallow indentation before any formation of pile-up. This clearly shows its extended applicability not only for soft metals but also for hard metals whose elastic deformation predominates with no tendency for piling up, and also for the case of purely elastic materials when $\left(a_{\mathrm{c}} / a_{\mathrm{o}}\right)^{2}=0.5$.

As pointed out in Section 6, the application of Eq. (14) to the indentation data, that is, the $P-h$ curves of a material whose post-yield behaviour is not known, can be achieved through an iterative process. The dependence of the correction factor on the indentation depth allows this process to succeed after several iterations each consisting of the following steps:

(a) The unloading portion of the $P-h$ curve in every cycle is fitted with the non-linear function given by Eq. (8) so that it could be extrapolated to the zero load axis thus yielding the residual indentation depth, hence the ratio $h_{\mathrm{r}} / h_{\mathrm{t}}$.

(b) The projected area radius $a_{\mathrm{o}}$, corresponding to $h_{\mathrm{t}}$ is calculated for every cycle. Thus initial values of Meyer hardness and representative strain are obtained assuming $a_{\mathrm{o}}$ to be the contact area radius.

(c) The strain hardening exponent $n$ is determined by regression analysis of the hardness versus representative strain plot on a logarithmic scale. 
(d) From the knowledge of the initial value of $n, c^{2}$ is calculated from Eq. (11) and substituted together with the ratio $\left(h_{\mathrm{r}} / h_{\mathrm{t}}\right)$ into Eq. (14) to determine an initial value for the true contact area radius $a_{\mathrm{c}}$, at every loading cycle. Thus Meyer hardness and representative strain corresponding to $a_{\mathrm{c}}$ are obtained.

(e) Then $n$ is determined in the same manner as in step (c), based on the new $a_{\mathrm{c}}$, and is compared with the input value for $n$ used to calculate $a_{\mathrm{c}}$ in step (d). If the difference between the two values is larger than a predefined tolerance, then steps (d) and (e) are repeated, otherwise the iteration process terminates.

The proposed new empirical relation equation (14) was applied to the $P-h$ curves produced by FE simulation of indentation for the materials S3, S5, and SS. The flow stress curve generated by this method, the one obtained in Section 3 using the contact radius from the simulated indentation profile as well as that produced in Section 4 by the conventional characterisation method that ignores the pile-up effect can be compared with the original input stress-strain curve for the three materials by referring to Figs. 13-15. Included in the plots is also the resulting $n$

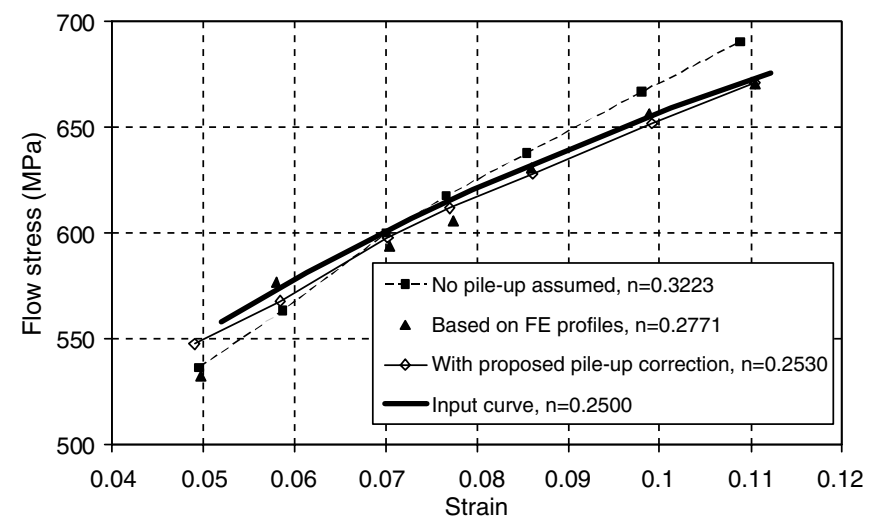

Fig. 13. Predicted and original flow stress curves for the stainless steel material.

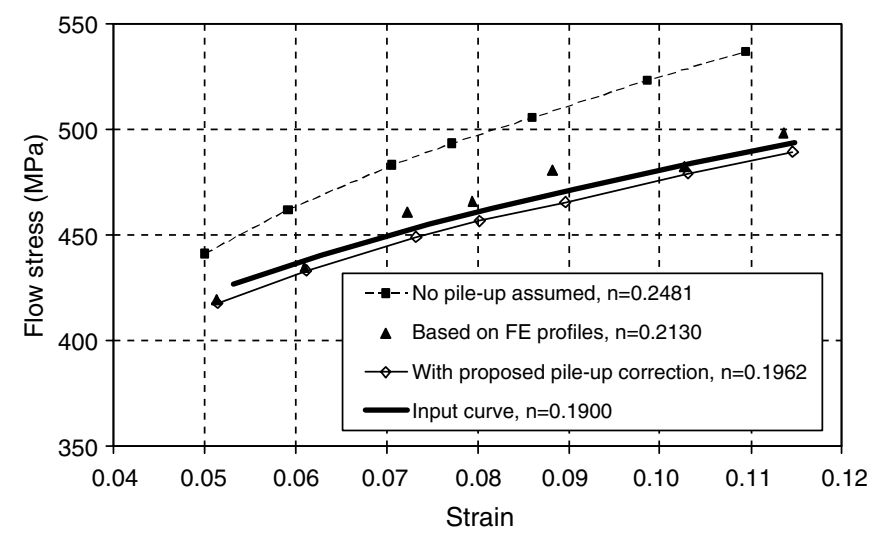

Fig. 14. Predicted and original stress-strain curves for material S3.

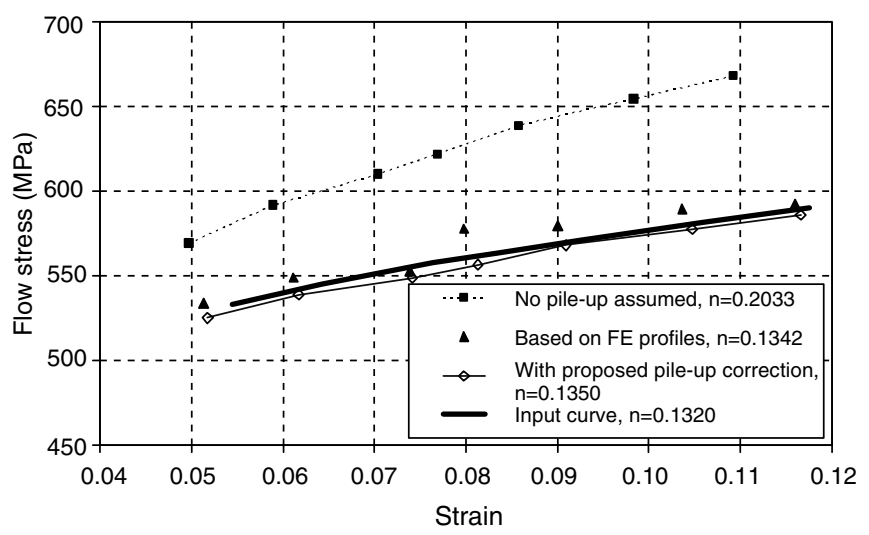

Fig. 15. Predicted and original flow stress curves for material S5.

Table 5

Predicted and original values of strain hardening exponent and yield stress for the three materials studied

\begin{tabular}{|c|c|c|c|c|c|}
\hline \multirow{2}{*}{$\begin{array}{l}\text { Type of } \\
\text { idealised } \\
\text { material }\end{array}$} & \multirow{2}{*}{$\begin{array}{l}n \text { and } \sigma_{0.2} \\
(\mathrm{MPa})\end{array}$} & \multirow{2}{*}{$\begin{array}{l}\text { Input } \\
\text { curve }\end{array}$} & \multicolumn{3}{|c|}{ Characterisation predictions } \\
\hline & & & $\begin{array}{l}\text { No pile-up } \\
\text { correction }\end{array}$ & $\begin{array}{l}\text { Contact radius } \\
\text { from finite } \\
\text { element } \\
\text { simulation }\end{array}$ & $\begin{array}{l}\text { Pile-up } \\
\text { correction }\end{array}$ \\
\hline \multirow[t]{2}{*}{ S3 } & $n$ & 0.19 & 0.25 & 0.21 & 0.20 \\
\hline & $\sigma_{0.2}$ & 250 & 221 & 232 & 242 \\
\hline \multirow[t]{2}{*}{ S5 } & $n$ & 0.13 & 0.20 & 0.13 & 0.13 \\
\hline & $\sigma_{0.2}$ & 375 & 334 & 377 & 369 \\
\hline \multirow{2}{*}{$\begin{array}{c}\text { Stainless } \\
\text { steel }\end{array}$} & $n$ & 0.25 & 0.32 & 0.28 & 0.25 \\
\hline & $\sigma_{0.2}$ & 285 & 221 & 257 & 280 \\
\hline
\end{tabular}

value for every curve. These values for $n$ together with the respective values for the $0.2 \%$ yield stress are listed in Table 5 for each curve.

As observed in Figs. 13-15, the proposed method based on Eq. (14) and an iterative procedure have proved to be very effective in accounting for the pile-up effect allowing the determination of the true contact area, and thus the stress-strain curve. As Table 5 shows, the predicted strain hardening exponent and $0.2 \%$ yield stress are within $3 \%$ of the corresponding values of the respective input curve.

\section{Conclusions}

A modified correction formula has been proposed for assessing the pile-up effect on indentation data which leads to a more reliable post-yield characterisation of structural steels through an iterative algorithm. It was shown in Section 6 that, for relative large characteristic strains, the pile-up parameter $c$ predicted by finite element modelling approaches a constant value. Existing pile-up correction expressions may have been fitted to indentation data corresponding to such strains. In contrast, the expression for $c$ proposed here accounts for its variation with indentation depth. 
The development of the method was based on finite element simulations of indentations on materials whose post-yield stress-strain curve had been simplified and characterised as a single power law function. This allowed a systematic assessment of the procedure proposed so that it can be adopted for the analysis of experimental indentation data obtained under comparable conditions. An important feature in the simulation was to take into account friction between the contact surfaces, which proved to be essential in modelling the accurate indentation response of a material that piles up.

Although the properties of the three materials, on which the development of empirical equation (14) was based, cover a realistic range of values for structural steel, there is scope for further assessment and, possibly, refinement of the pile-up correction so that the proposed methodology could be applied more widely and with confidence. It should be borne in mind that the latter is applicable to materials without a yield plateau, that can be characterised by a yield stress, a strain hardening exponent, and a constant constraint factor $C$. A more complex material behaviour would require a more advanced characterisation tool.

\section{References}

[1] Tabor D. The hardness of metals. Oxford: Clarendon Press; 1951. p. 69.

[2] Field JS, Swain MV. J Mater Res 1995;10:101.

[3] Bolshakov A, Pharr GM. J Mater Res 1998;13:1049.

[4] Matthews JR. Acta Metall 1980;28:311.

[5] Hill R. J Mech Phys Solids 1987;35:565.

[6] Taljat B, Zacharia T, Kosel F. Int J Solids Struct 1998;35: 4411.

[7] ANSYS University Research, v. 8.1. Canonsburg (PA): ANSYS, Inc.; 2005. Available from: www.ansys.com.

[8] Sneddon IN. Int J Eng Sci 1965;3:47.

[9] Tabor D. Proc R Soc Lond A: Math Phys Sci 1948;192: 247.

[10] Cao YP, Lu J. Acta Mater 2004;52:4023.

[11] Yurkov AL, Skvortsov VN, Buyanovsky IA, Matvievsky RM. J Mater Sci Lett 1997;16:1370.

[12] Lide DR. Handbook of chemistry and physics. Boca Raton (FL): CRC Press; 1995.

[13] Bucaille JL, Stauss S, Schwaller P, Michler J. Thin Solid Films 2004;447:239.

[14] Field JS, Swain MV. J Mater Res 1993;8:297.

[15] Norbury AL, Samuel T. J Iron Steel Inst 1928;17:673.

[16] Hill R, Storakers B, Zdunek AB. Proc R Soc Lond A: Math Phys Eng Sci 1989;423:301. 